\title{
Clinical and radiological results of indirect decompression after anterior lumbar interbody fusion in central spinal canal stenosis
}

\author{
*Dong Hyun Lee, MD,' Dong-Geun Lee, MD,1 Jin Sub Hwang, MD,' Jae-Won Jang, MD, PhD,1 \\ Dae Hyeon Maeng, MD, ${ }^{2}$ and Choon Keun Park, MD, PhD ${ }^{1}$
} 'Department of Neurosurgery, Spine Center, The Leon Wiltse Memorial Hospital; and 2Department of Cardiovascular Surgery,
The Leon Wiltse Memorial Hospital, Suwon, Gyeonggi-do, South Korea

\begin{abstract}
OBJECTIVE Whereas the benefits of indirect decompression after lateral lumbar interbody fusion are well known, the effects of anterior lumbar interbody fusion (ALIF) have not yet been verified. The purpose of this study was to evaluate the clinical and radiological effects of indirect decompression after ALIF for central spinal canal stenosis. In this report, along with the many advantages of the anterior approach, the authors share cases with good outcomes that they have encountered.
\end{abstract}

METHODS The authors performed a retrospective analysis of 64 consecutive patients who underwent ALIF for central spinal canal stenosis with instability and mixed foraminal stenosis between January 2015 and December 2018 at their hospital. Clinical assessments were performed using the visual analog scale score, the Oswestry Disability Index, and the modified Macnab criteria. The radiographic parameters were determined from pre- and postoperative cross-sectional MRI scans of the spinal canal and were compared to evaluate neural decompression after ALIF. The average follow-up period was $23.3 \pm 1.3$ months.

RESULTS All clinical parameters, including the visual analog scale score, Oswestry Disability Index, and modified Macnab criteria, improved significantly. The mean operative duration was $254.8 \pm 60.8$ minutes, and the intraoperative bleeding volume was $179.8 \pm 119.3 \mathrm{ml}$. In the radiological evaluation, radiological parameters of the cross-sections of the spinal canal showed substantial development. The spinal canal size improved by an average of $43.3 \%(p<0.001)$ after surgery. No major complications occurred; however, aspiration guided by ultrasonography was performed in 2 patients because of a pseudocyst and fluid collection.

CONCLUSIONS ALIF can serve as a suitable alternative to extensive posterior approaches. The authors suggest that ALIF can be used for decompression in central spinal canal stenosis as well as restoration of the foraminal dimensions, thus allowing decompression of the nerve roots.

https://thejns.org/doi/abs/10.3171/2020.7.SPINE191335

KEYWORDS anterior lumbar interbody fusion; ALIF; degenerative disc disease; foramen measurement; central spinal canal measurement; indirect decompression

工 UMBAR surgery has become an alternative strategy for use in patients who do not respond to conservative treatment. Among the many surgical choices, spinal fusion plays an important role in addressing debilitating low-back and leg pain related to continued abnormal motion of the affected segments. ${ }^{1,2}$ Whereas the benefits of indirect decompression after lateral lumbar interbody fusion are well known, ${ }^{3-7}$ and although several studies have demonstrated significant indirect foraminal decom- pression after anterior lumbar interbody fusion (ALIF), ${ }^{8-11}$ little is known about the results of indirect decompression of the central spinal canal after ALIF. There have been no studies on the degree of decompression resulting from indirect decompression without posterior decompression in central spinal canal stenosis. The purpose of this study was to evaluate the clinical and radiological effects of indirect decompression after ALIF for central spinal canal stenosis.

ABBREVIATIONS ALIF = anterior lumbar interbody fusion; DSCSA = dural sac cross-sectional area; ODI = Oswestry Disability Index; VAS = visual analog scale SUBMITTED November 13, 2019. ACCEPTED July 27, 2020.

INCLUDE WHEN CITING Published online January 15, 2021; DOI: 10.3171/2020.7.SPINE191335.

${ }^{*}$ D.H.L. and D.G.L. contributed equally to this work. 

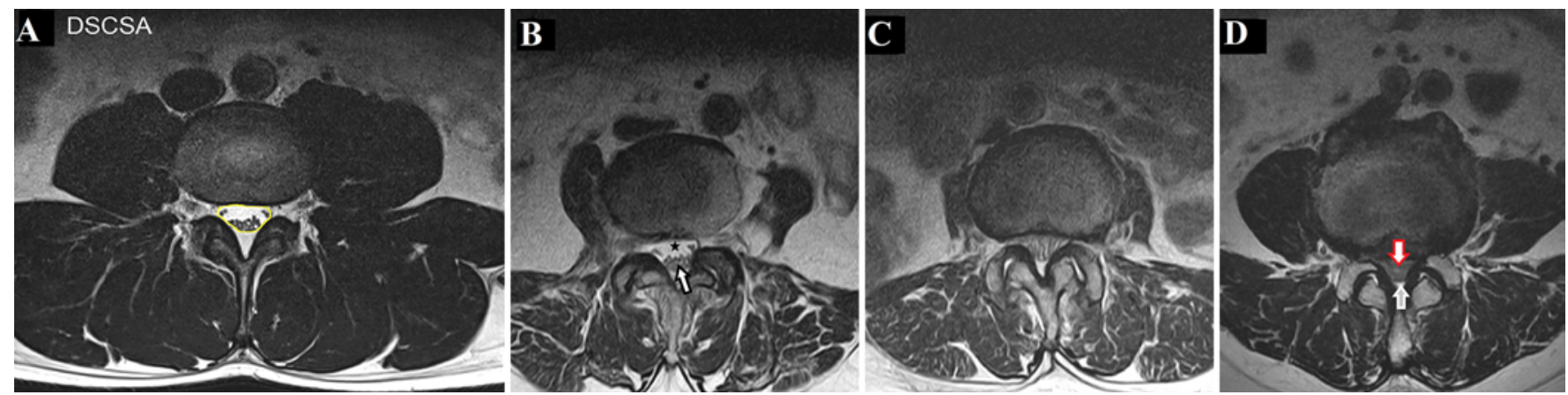

FIG. 1. The MRI grade is dependent on the morphology and density of the rootlets in the CSF, as seen on axial images. A: The DSCSA was measured based on axial MRI scans. B: Grade A (no or minor stenosis): the CSF is clearly visible inside the dural sac (star), but rootlet distribution is inhomogeneous (arrow). C: Grade B (moderate stenosis): the rootlets occupy the whole of the dural sac, but they can still be individually identified. D: Grade C (severe stenosis): the dural sac presents only a homogeneous gray signal with no CSF signal visible (red arrow), and the rootlets cannot be recognized. There is epidural fat present posteriorly (white arrow). Figure is available in color online only.

\section{Methods}

\section{Study Subjects}

We performed a retrospective analysis of 64 consecutive patients who underwent ALIF for central spinal canal stenosis with instability and mixed foraminal stenosis and who were treated by the same team of spinal and vascular surgeons in our hospital between January 2015 and December 2018. The indications included radiculopathy, neurological deficits, and/or severe low-back pain with radiological evidence of recurrent disc herniation, foraminal stenosis, or degenerative disc disease, along with a lack of symptom resolution with conservative treatment.

Patients with a history of posterior fusion or laminectomy and less than 12 months of postoperative radiological or clinical follow-up data were excluded. The ALIF procedure was performed with a standard minimally invasive retroperitoneal approach for removal of the anterior longitudinal ligament, discectomy, and placement of a polyetheretherketone interbody implant of an appropriate height and for appropriate lordosis with a percutaneous pedicle screw system at the interbody fusion level. Cages filled with allograft bone chips and bone morphogenetic protein-2 were placed into the intervertebral spaces, and the remaining space was filled with allograft bone chips.

We used the dural sac cross-sectional area (DSCSA) as a measure of lumbar central spinal canal stenosis. Radiographic parameters were determined using MRI scans reflecting DSCSA of the spinal canal and were compared preoperatively, on postoperative day 2 , and at the last follow-up to evaluate neural decompression after ALIF. The average follow-up period was $23.3 \pm 1.3$ months, and the average follow-up period for the last MRI session was 20.8 \pm 3.1 months after surgery.

This investigation was conducted in accordance with our agency guidelines, which comply with international laws and policies, and was approved by The Leon Wiltse Memorial Hospital Institutional Review Board.

\section{Evaluation of the Radiological and Clinical Results}

Radiological data were compared based on the preoperative and latest follow-up spine MRI scans. We analyzed the diagnosis, operative level(s), and central spinal canal dural diameter on the radiological images. T2-weighted midsagittal MRI scans were used to obtain standardized central spinal canal measurements based on the DSCSA. Single slices through the disc center were obtained on the axial view to measure and compare the DSCSA under the same conditions. The outer border of the dura mater was manually traced on the selected axial slice, and the enclosed area was measured as the DSCSA. The extent of decompression was calculated according to the percent increase in the DSCSA, as follows: (last follow-up DSCSA - preoperative DSCSA)/preoperative DSCSA $\times 100 \% .4,6$ All measurements were made digitally using computer software (Fig. 1).

The grade of spinal stenosis was evaluated using the MRI scans according to Schizas' MRI grading scale, ${ }^{13}$ which has previously been used for the precise evaluation of the degree of indirect neural decompression after lateral lumbar interbody fusion; ${ }^{12}$ this grade depends on the morphology and density of rootlets in the CSF, as observed in an axial image. Schizas' MRI grading scale is as follows: grade A (no or minor stenosis) - there is CSF clearly visible inside the dural sac, but its distribution is inhomogeneous; grade B (moderate stenosis) - rootlets occupy the whole of the dural sac, but they can still be individually identified; and grade $\mathrm{C}$ (severe stenosis) - the dural sac presents only a homogeneous gray signal with no CSF signal visible, and rootlets cannot be recognized (Fig. 1). Preoperative, postoperative, and last follow-up values (DSCSA, lordosis angle, and disc height) were measured by three inspectors who were blinded to this study. The values were determined using the average of the values obtained by the three inspectors. The Schizas' MRI grading scale was also evaluated one time by three inspectors, and the final decision grade was obtained when two or more of the same grades were received. There were no cases with three different grades (grades A, B, and C). The interrater agreement of the stenosis grade was calculated using Fleiss kappa ${ }^{14}$ coefficients and R 3.1.2 for Windows software.

Additionally, we investigated the relationship between radiological factors and DSCSA. The efficacy outcomes, including the visual analog scale (VAS) scores for back 
TABLE 1. Summary of demographic and diagnostic data in patients with central spinal canal stenosis

\begin{tabular}{lc}
\hline \multicolumn{1}{c}{ Group } & Total \\
\hline No. of patients & 64 \\
\hline No. of spinal fusion levels & 123 \\
\hline Sex ratio (F/M) & $48: 16$ \\
\hline Age in yrs (mean \pm SD) & $67.8 \pm 8.5$ \\
\hline Diagnosis (no. of patients) & 17 \\
\hline Spondylolisthesis & 19 \\
\hline Degenerative disc disease & 48 \\
\hline Foraminal stenosis & \\
\hline No. of fusion levels & 21 \\
\hline 1 & 30 \\
\hline 2 & 10 \\
\hline 3 & 3 \\
\hline 4 & \\
\hline Spinal fusion level & 0 \\
\hline L1/2 & 3 \\
\hline L2/3 & 24 \\
\hline L3/4 & 55 \\
\hline L4/5 & 41 \\
\hline L5/S1 & 64 \\
\hline Percutaneous pedicle screw fixation (no. of patients) \\
\hline
\end{tabular}

and leg pain, ${ }^{15}$ the Oswestry Disability Index (ODI),${ }^{16}$ and the modified Macnab criteria, ${ }^{17}$ were measured preoperatively, postoperatively, and at the last follow-up. The modified Macnab criteria were applied to evaluate the surgical outcomes: excellent indicates free of pain, allowing the patient to work normally; good indicates occasional radicular pain, slight activity limitation that does not influence work or quality of life; fair indicates some improved functional capacity, still handicapped or unemployed; and poor indicates no improvement. All patients who had undergone surgery were followed for at least 12 months. The clinical parameters investigated included the incidence of complications (e.g., cage subsidence, vertebral fracture, dural tear, incomplete decompression, reoperation, incidental durotomy, epidural hematoma, headache, infection, recurrence rate, major vessel injury, peritoneal injury, and urinary injury); the intraoperative bleeding volume; and the operative duration.

\section{Statistical Analysis}

$\mathrm{R}$ 3.1.2 for Windows software was used to perform the Wilcoxon rank-sum test, multivariate regression, and Pearson coefficient test. When the $\mathrm{p}$ value was $<0.05$, the result was judged to be statistically significant.

\section{Results}

A total of 64 patients comprised 48 women and 16 men ranging in age from 49 to 84 years (mean $67.8 \pm 8.5$ years). The average follow-up duration was $23.3 \pm 1.3$ months, and ALIF was performed at 123 levels. The intraopera-

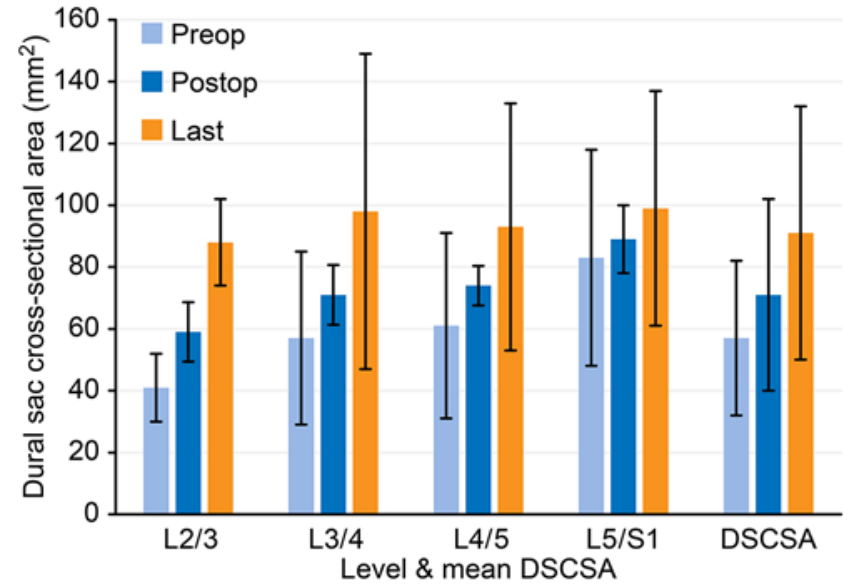

FIG. 2. Bar graph showing a comparison of DSCSA by level and the mean DSCSA (except preoperative grade A) between the preoperative and postoperative MRI. Figure is available in color online only.

tive bleeding volume was $179.8 \pm 119.3 \mathrm{ml}$, and the mean operative duration was $254.8 \pm 60.8$ minutes. The demographic characteristics of the patients, including the preoperative diagnoses, are summarized in Table 1.

\section{Radiographic and Clinical Outcomes}

During the radiological evaluation, significant expansion of the DSCSA was measured. The mean DSCSA increased significantly by $16.4 \%$ immediately after surgery and $43.3 \%$ at the last follow-up ( $<<0.001)$. There was more expansion in the follow-up period than immediately after surgery. Except for preoperative grade A, which did not require central spinal canal decompression according to Schizas' MRI grading scale, the mean DSCSA increased significantly by $24.6 \%$ immediately after ALIF and by $59.6 \%(\mathrm{p}<0.001)$ at the last follow-up (Fig. 2). At each fusion level, the mean DSCSA increased significantly by $43.9 \%, 24.6 \%, 21.3 \%$, and $7.2 \%$ after surgery. There were greater expansion rates of $114.6 \%, 71.9 \%, 52.5 \%$, and $19.3 \%$ at the L2/3, L3/4, L4/5, and L5/S1 levels, respectively, at the last follow-up (L2/3: $\mathrm{p}=0.008 ; \mathrm{L} 3 / 4, \mathrm{~L} 4 / 5$, L5/S1: p < 0.001; Fig. 2).

Spinal stenosis classified via MRI revealed grade C stenosis at 52 levels, grade B at 46 levels, and grade A at 25 levels. Other than the L5/S1 level, only 3 of the L4/5 levels were considered grade A. Of the 52 levels considered grade $\mathrm{C}$ preoperatively, 10 improved to grade $\mathrm{A}, 28$ improved to grade $\mathrm{B}$, and 14 remained at grade $\mathrm{C}$ postoperatively. Of the 46 levels considered grade B preoperatively, 27 improved to grade A, and 19 remained at grade $\mathrm{B}$. There were no cases of worsening to grade $\mathrm{C}$. All 25 levels considered grade A remained at grade A. Consequently, the MRI grade did not worsen at any level. Among the levels that were grade $\mathrm{C}$ preoperatively and remained grade C postoperatively, DSCSA increased by $30.6 \%$, and among those that were grade $\mathrm{B}$ preoperatively and remained grade B postoperatively, DSCSA increased by $21.5 \%$. An increase of $11.7 \%$ was also observed among the levels that were grade A preoperatively, with no or minor central spinal canal stenosis. 
TABLE 2. Association between radiological factors and improvement of canal size

\begin{tabular}{|c|c|c|c|c|c|c|}
\hline \multirow[b]{2}{*}{ Variable } & \multicolumn{3}{|c|}{ Canal Size (postop - preop) } & \multicolumn{3}{|c|}{ Canal Size (last FU - preop) } \\
\hline & $p$ Value & Pearson Correlation & $\begin{array}{c}\text { Pearson Correlation } \\
\text { p Value }\end{array}$ & $p$ Value & Pearson Correlation & $\begin{array}{c}\text { Pearson Correlation } \\
\text { p Value }\end{array}$ \\
\hline Disc height (postop - preop) & 0.724 & -0.062 & 0.500 & 0.199 & 0.047 & 0.606 \\
\hline Disc height (last FU - preop) & 0.566 & -0.52 & 0.568 & 0.296 & -0.055 & 0.551 \\
\hline Lordosis (postop - preop) & 0.789 & -0.015 & 0.875 & 0.937 & -0.061 & 0.519 \\
\hline Lordosis (last FU - preop) & 0.843 & -0.038 & 0.687 & 0.357 & -0.081 & 0.390 \\
\hline Cage height (mm) & 0.214 & -0.034 & 0.734 & 0.682 & 0.081 & 0.425 \\
\hline Cage angle $\left(^{\circ}\right)$ & 0.399 & -0.199 & $0.049^{*}$ & 0.208 & -0.251 & $0.013^{*}$ \\
\hline Preop grade $B$ & $0.024 \dagger$ & & & $0.042 \dagger$ & & \\
\hline Preop grade $\mathrm{C}$ & $0.001 \dagger$ & & & $0.001 \dagger$ & & \\
\hline
\end{tabular}

FU $=$ follow-up.

* Statistical significance was determined using the Pearson correlation coefficient.

† Statistical significance was determined using multivariate regression analysis.

Concordance of the preoperative Schizas' stenosis grade kappa results was 0.898 (95\% CI 0.824-0.973; p < 0.005 ), and the coincidence was very good. According to the interrater agreement for each preoperative grade, the kappa value of grade A was 0.900 , that of grade B was 0.861 , and that of grade $\mathrm{C}$ was 0.933 ( $\mathrm{p}<0.005$ ). Each grade coincidence was very good. Concordance of the last follow-up Schizas' stenosis grade kappa results was 0.869 (95\% CI $0.788-0.950 ; p<0.005$ ), and the coincidence was very good. According to the interrater agreement for each last follow-up grade, the kappa value of grade A was 0.943 , that of grade B was 0.898 , and that of grade $\mathrm{C}$ was $0.923(\mathrm{p}<0.005)$. Each grade coincidence was very good.

In the association between radiological factors and postoperative central cross-sectional area, grades B and C significantly improved in DSCSA compared with grade A. There was no significant correlation between the size of the implant and stenosis improvement (Table 2). Additionally, we studied the relationship between the implant and radiographic assessments, including disc height, lordosis, and canal size improvement. More to the point is the fact that there was a significant correlation between the lordotic angle of implant and DSCSA (Tables 2 and 3). Lordosis significantly improved after surgery; however, there was no significant correlation between lordosis and stenosis improvement (Fig. 3). Regarding the clinical outcomes, the average follow-up duration was $23.3 \pm 1.3$ months. The mean preoperative VAS score for low-back pain was 7.73 \pm 1.41 , and at the last follow-up the score improved to 3.84 \pm 1.48 ( $\mathrm{p}<0.001)$. The mean preoperative VAS score for leg pain was $8.16 \pm 1.18$, and it improved to $3.83 \pm 1.47$ postoperatively $(\mathrm{p}<0.001)$. The mean preoperative ODI was $26.53 \pm 3.13$, which decreased to $4.84 \pm 2.26$ postoperatively ( $p<0.001)$. There were no cases of worsening of the patient's condition. All clinical outcome scores significantly improved postoperatively.

At the last follow-up, patient satisfaction with the surgery was evaluated with the modified Macnab criteria. The rate of excellent and good patient satisfaction was found to be $81.25 \%$, and the rate of fair patient satisfaction was $18.75 \%$. None of the patients experienced serious complications associated with the surgery. Transient leg numbness occurred in 2 patients because of a pseudocyst and fluid collection, and aspiration guided by ultrasonography was performed. We were able to avoid several major concerns associated with ALIF, including significant vascular injuries, retrograde ejaculation, urological complications, and abdominal muscle damage, through careful patient selection and access to a highly experienced and well-trained surgeon.

\section{Illustrative Cases \\ Case 1}

A 59-year-old man had low-back pain, radicular leg pain, and intermittent claudication. MRI showed severe central spinal canal stenosis from L2 to L5 with neural foraminal stenosis at $\mathrm{L} 4 / 5$ and $\mathrm{L} 5 / \mathrm{S} 1$. The preoperative

TABLE 3. Relationship between implant and radiographic factors

\begin{tabular}{ccccrrrr}
\hline \multirow{2}{*}{ Variable } & Correlation & $\begin{array}{c}\text { Disc Height } \\
\text { (postop-preop) }\end{array}$ & $\begin{array}{c}\text { Disc Height } \\
\text { (last FU - preop) }\end{array}$ & $\begin{array}{c}\text { Lordosis } \\
\text { (postop - preop) }\end{array}$ & $\begin{array}{c}\text { Lordosis } \\
\text { (last FU - preop) }\end{array}$ & $\begin{array}{c}\text { Canal Size } \\
\text { (postop - preop) }\end{array}$ & $\begin{array}{c}\text { Canal Size } \\
\text { (last FU - preop) }\end{array}$ \\
\hline \multirow{2}{*}{ Cage height } & Pearson correlation & -0.105 & -0.174 & -0.110 & -0.010 & -0.034 & 0.081 \\
\cline { 2 - 8 } & p value & 0.299 & 0.084 & 0.284 & 0.919 & 0.734 & 0.425 \\
\hline \multirow{2}{*}{ Cage angle } & Pearson correlation & -0.101 & 0.017 & -0.037 & -0.077 & -0.199 & -0.251 \\
\cline { 2 - 8 } & p value & 0.323 & 0.867 & 0.722 & 0.455 & $0.049^{*}$ & $0.013^{*}$ \\
\hline
\end{tabular}

\footnotetext{
* Statistical significance was determined using the Pearson correlation coefficient.
} 
DSCSA assessed via MRI was $30 \mathrm{~mm}^{2}$ at the $\mathrm{L} 2 / 3$ level, $17 \mathrm{~mm}^{2}$ at the L3/4 level, $22 \mathrm{~mm}^{2}$ at the L4/5 level, and $65 \mathrm{~mm}^{2}$ at the L5/S1 level. These levels were preoperative MRI grade $\mathrm{C}$ except for the L5/S1 level, which was grade A. Four-level ALIF was performed. Cages measuring 12 $\mathrm{mm}$ in height, $23 \mathrm{~mm}$ in depth, and $31 \mathrm{~mm}$ in width with a lordotic angle of $8^{\circ}$ were used in the intervertebral spaces from L2 to L5, and a cage measuring $12 \mathrm{~mm}$ in height, 27 $\mathrm{mm}$ in depth, and $35 \mathrm{~mm}$ in width with a lordotic angle of $8^{\circ}$ was used in the intervertebral space at the L5/S1 level with allograft bone. The last follow-up was at 14 months postoperatively. Follow-up images revealed successful indirect central decompression after ALIF, with stretching and straightening of the relaxed ligamentum flavum. The last follow-up DSCSA was $100 \mathrm{~mm}^{2}$ at the L2/3 level, 70 $\mathrm{mm}^{2}$ at the L3/4 level, $94 \mathrm{~mm}^{2}$ at the L4/5 level, and 85 $\mathrm{mm}^{2}$ at the L5/S1 level. At the last follow-up, the level of central decompression was grade B at the L2/3 and L3/4 levels and grade $\mathrm{A}$ at the L4/5 and L5/S1 levels. The extent of decompression was $233.3 \%$ at the L2/3 level, $311.8 \%$ at the L3/4 level, $327.3 \%$ at the L4/5 level, and 30.8\% at the L5/S1 level. A percutaneous pedicle screw system was used. Posterior direct decompression was not performed. The mean VAS score for low-back pain improved from 9 preoperatively to 2 at the last follow-up. The mean VAS score for leg pain improved from 9 preoperatively to 3 at the last follow-up. The mean ODI improved from 28 preoperatively to 3 at the last follow-up (Fig. 4).

\section{Case 2}

A 66-year-old woman had numbness and radicular pain in both lower extremities, back pain, and intermittent claudication. Preoperative MRI showed severe spinal canal stenosis with a herniated disc at L3/4 and L4/5 and neural foraminal stenosis at L5/S1. At L3/4, the preoperative DSCSA was $50 \mathrm{~mm}^{2}$ and the MRI grade was C. At L4/5, the preoperative DSCSA was $48 \mathrm{~mm}^{2}$ and the MRI grade was C. At L5/S1, the preoperative DSCSA was 112 $\mathrm{mm}^{2}$ and the MRI grade was A. Three-level ALIF was performed. Cages measuring $11 \mathrm{~mm}$ in height, $27 \mathrm{~mm}$ in depth, and $35 \mathrm{~mm}$ in width with a lordotic angle of $8^{\circ}$ were placed in the intervertebral spaces from L3 to L5, and a cage measuring $12 \mathrm{~mm}$ in height, $27 \mathrm{~mm}$ in depth, and 35 $\mathrm{mm}$ in width with a lordotic angle of $8^{\circ}$ was placed at the L5/S1 level with allograft bone. The last follow-up was at 13 months postoperatively. The last follow-up DSCSA was $182 \mathrm{~mm}^{2}$ at the L3/4 level, $158 \mathrm{~mm}^{2}$ at the L4/5 level, and $134 \mathrm{~mm}^{2}$ at the L5/S1 level. At the last follow-up, the central spinal canal stenosis showed improvement from grade $\mathrm{C}$ to grade A at the L3/4 and L4/5 levels. At L5/S1, the grade A was not changed. The extent of decompression after surgery was $264 \%$ at the L3/4 level, $229.2 \%$ at the L4/5 level, and $19.6 \%$ at the $\mathrm{L} 5 / \mathrm{S} 1$ level. A percutaneous pedicle screw system was used for posterior spinal fixation. Sufficient indirect decompression of the central spinal canal was achieved without any additional decompression. The mean VAS score for low-back pain improved from 8 preoperatively to 4 at the last follow-up, and the mean VAS score for leg pain improved from 9 preoperatively to 3 at the last follow-up. The mean ODI improved from 27 preoperatively to 4 at the last follow-up (Fig. 5).

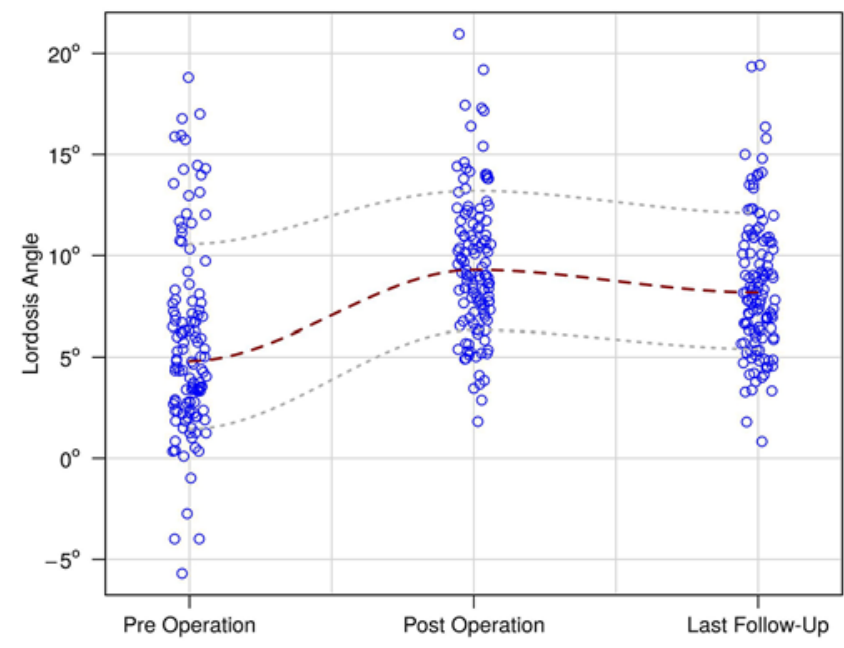

FIG. 3. Scatterplot showing the change in lordosis from preoperative through postoperative and last follow-up MRI. A locally weighted scatterplot smoothing (LOWESS) line is shown in dark red, with the $95 \% \mathrm{Cl}$ lines in light gray. Figure is available in color online only.

\section{Discussion}

The effect of ALIF is well known, especially in degenerative disc disease associated with discogenic pain and revision for failed posterior fusion. ${ }^{18}$ There are several reasons for the benefits of the ALIF approach; first, this technique provides a direct midline view of the disc space and extensive lateral exposure of the vertebral bodies, which enables efficient disc space clearance with rapid endplate preparation. Furthermore, the anterior approach increases the feasible implant size and surface area to facilitate aggressive lordotic correction and foraminal height restoration. These factors make it possible to secure the disc space and increase fusion splicing. ${ }^{10,19-22}$ Some reports have shown high fusion success rates of 94\%-97\%, which are higher than the success rates of posterior lumbar interbody fusion and transforaminal lumbar interbody fusion, demonstrating that ALIF is an effective surgical method for interbody fusion and can yield reliable clinical results. ${ }^{1}$

The greatest potential of ALIF seems to lie in indirect decompression. ${ }^{23}$ Cutting the anterior longitudinal ligament allows for the insertion of a taller graft. The effect of indirect foraminal decompression via ALIF has been reported;10,19,24 for example, Cho et al..$^{24}$ reported a foraminal expansion rate of $43.3 \%$. Rao et al. ${ }^{25}$ also achieved excellent results in expanding the foraminal dimensions (area 67\%, height $21 \%$, and width $38 \%$ ). According to a retrospective radiographic analysis comparing ALIF with transforaminal lumbar interbody fusion, the former was more effective in restoring the foraminal height. ${ }^{19}$ ALIF also preserves the posterior spinal muscles and anterolateral psoas muscles, which may reduce postoperative pain and disability. ${ }^{21,26-28}$

Extreme lateral interbody fusion is also a good technique for indirect decompression. Most authors have reported that there were no differences between the ALIF and extreme lateral interbody fusion groups in clinical outcomes, complication rate, and fusion rate..$^{2,29-31}$ However, it seems that ALIF has merits for postoperative disc 

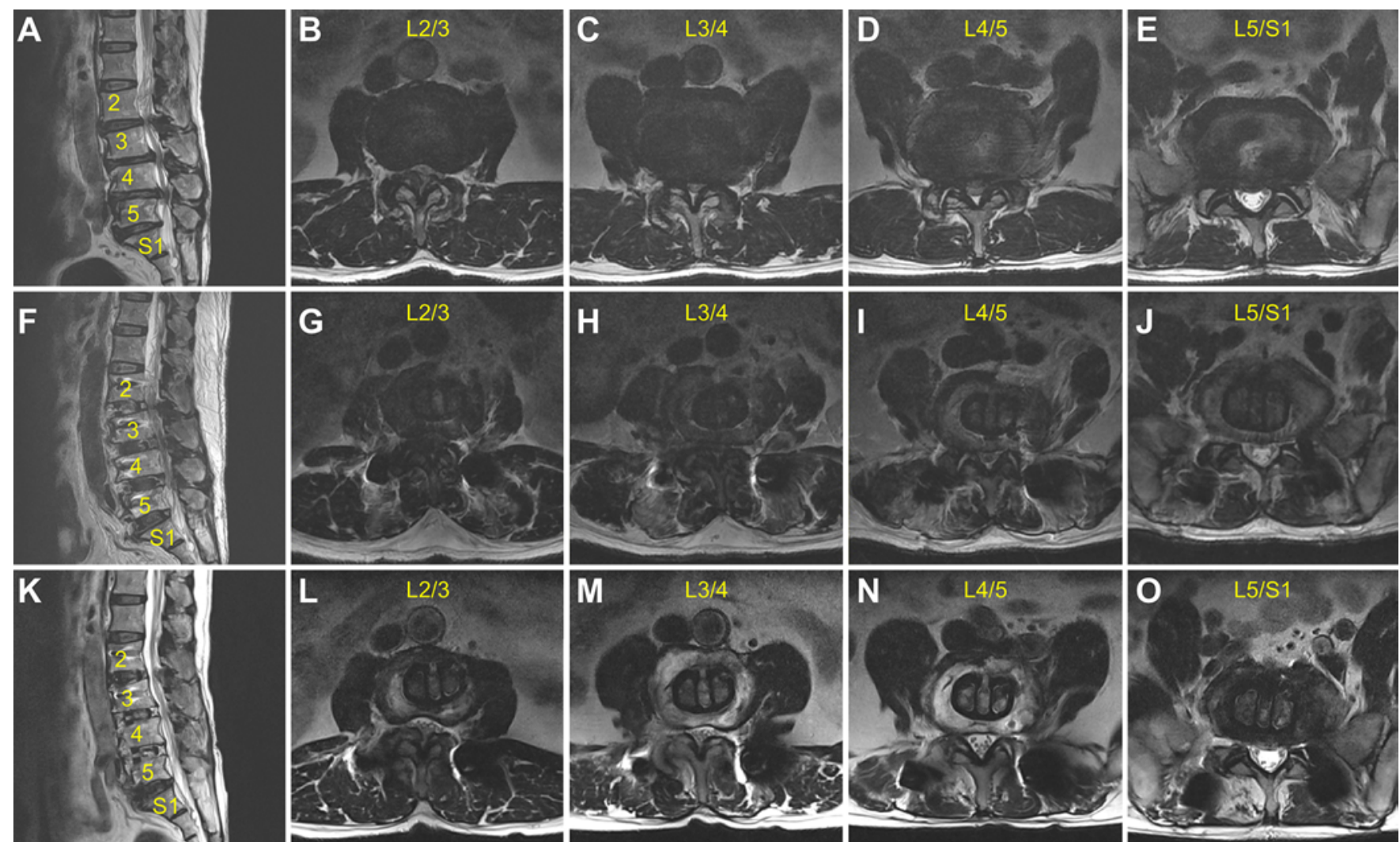

FIG. 4. Case 1. Sagittal and axial MRI scans obtained preoperatively, postoperatively, and at the 14-month follow-up revealing indirect decompression of the central spinal canal at L2/3, L3/4, L4/5, and L5/S1. Preoperative sagittal MRI scan (A) and axial MRI scans at the L2/3, L3/4, L4/5, and L5/S1 levels (B-E). The preoperative MRI grade was $C$ at the L2/3, L3/4, and L4/5 levels and $A$ at the L5/S1 level. Postoperative follow-up sagittal MRI scan (F) and axial MRI scans at the L2/3, L3/4, L4/5, and L5/S1 levels (G-J). Last follow-up sagittal MRI scan (K) and axial MRI scans at the L2/3, L3/4, L4/5, and L5/S1 levels (L-0). The last follow-up MRI grade was $B$ at the $L 2 / 3$ and $L 3 / 4$ levels and $A$ at the $L 4 / 5$ and $L 5 / S 1$ levels. Figure is available in color online only.

height and foraminal height, segmental and overall lumbar lordosis, and direct clearance of the disc space. ${ }^{1,25,32}$ Despite its many advantages, ALIF is still less preferred than other fusion methods for several reasons. One reason is doubt regarding indirect decompression for central spinal canal stenosis. Although many studies have reported the benefits of ALIF for indirect decompression of the foramen, indirect decompression of the central spinal canal has not been discussed. We considered how multilevel central spinal canal stenosis and foraminal stenosis could be effectively treated. ALIF is known as a good choice for treating foraminal stenosis, ${ }^{10,19,20,24}$ but what method should be applied for multilevel central stenosis with foraminal stenosis? Is treatment of the olisthetic level alone sufficient? Our study stems from the question of whether direct posterior decompression procedures are needed at all levels in multilevel central spinal canal stenosis that is suspected to be causing the patients' symptoms. The results of this study demonstrate that this approach is not necessary when ALIF is performed. We believe that the current study addresses these persisting doubts regarding indirect decompression for central spinal canal stenosis.

Direct decompression procedures are performed by resecting the impinging bone, ligaments, and disc material that are directly compressing the neural elements. ${ }^{33} \mathrm{Al}-$ though directly decompressing multiple spinal levels can sometimes be impossible or laborious, ALIF can produce sufficient indirect decompression. In this report, even a small extent of indirect central spinal canal decompression improved the patients' symptoms. Even patients in whom the MRI grade did not change at the operative level of the spine showed an improvement in the VAS score for back pain (from $7.45 \pm 1.64$ to $3.70 \pm 1.45$ ) and an improvement from $26.24 \pm 3.70$ to $5.33 \pm 2.63$ in the ODI. Because sufficient indirect decompression for central spinal canal stenosis was achieved without the need for additional direct decompression, we reduced the intraoperative blood loss volume and the operative duration, demonstrating that this procedure is efficient and requires less of the surgeon's time.

In our study, Schizas' grades B and C resulted in more stenosis improvement than grade A, suggesting that grade $\mathrm{C}$ is also quite improved by indirect decompression. There was no significant correlation between the implant size and stenosis improvement in our study. However, there was a significant effect of the lordotic angle of implants on the postoperative central cross-sectional area to obtain an improved central area. Cages with minimal lordotic angles distract the disc space more and indirectly open up the central canal to a greater degree than hyperlordotic 

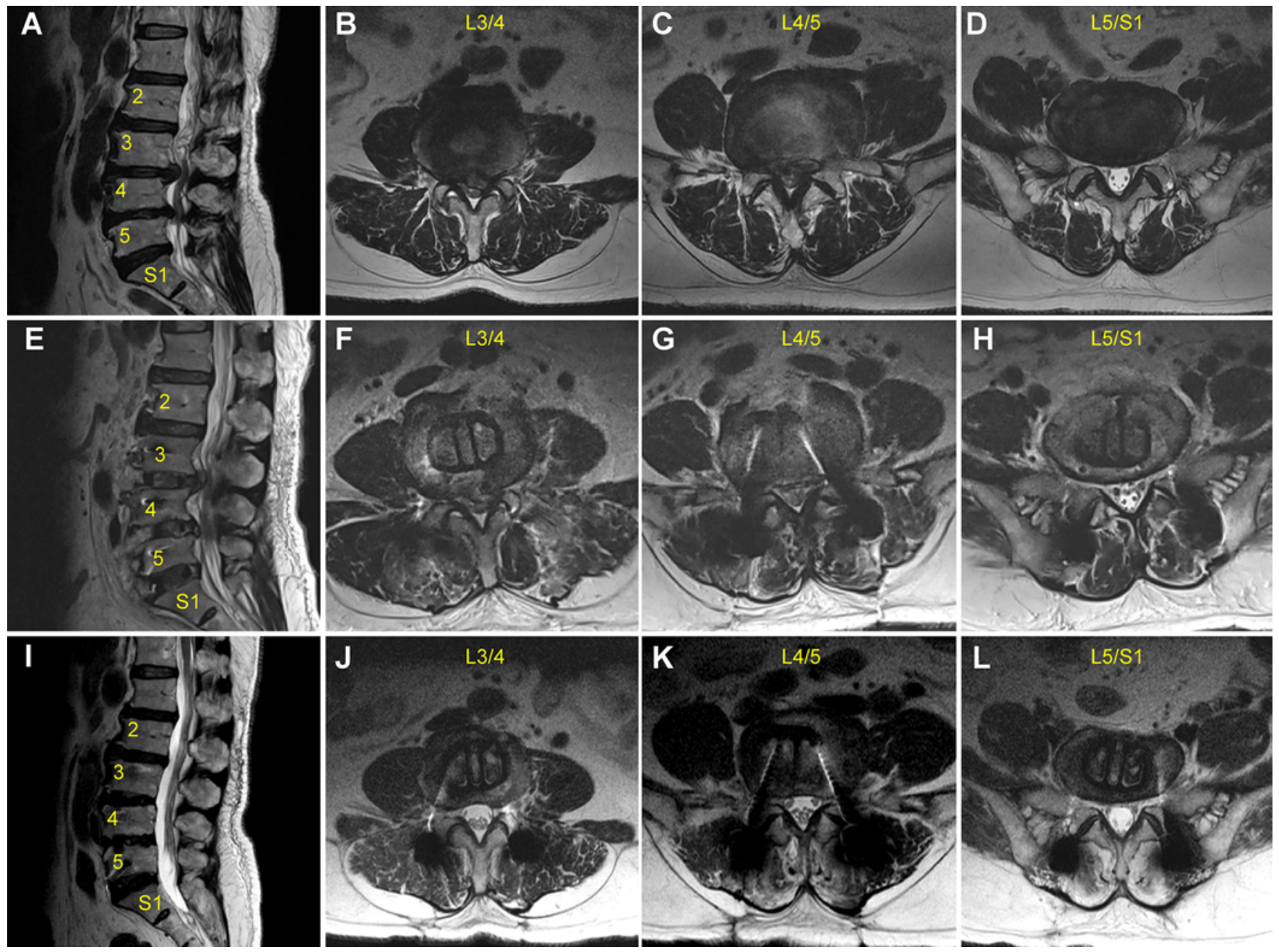

FIG. 5. Case 2. Sagittal and axial MRI scans obtained preoperatively, postoperatively, and at the 13-month follow-up revealing indirect decompression of the central spinal canal at the L3/4, L4/5, and L5/S1 levels. Preoperative sagittal MRI scan (A) and axial MRI scans at the $L 3 / 4, L 4 / 5$, and $L 5 / S 1$ levels (B-D). The preoperative MRI grade was $C$ at the $L 3 / 4$ and $L 4 / 5$ levels and $A$ at the L5/S1 level. Postoperative follow-up sagittal MRI scan (E) and axial MRI scans at the L3/4, L4/5, and L5/S1 levels (F-H). Last follow-up sagittal MRI scan (I) and axial MRI scans at the L3/4, L4/5, and L5/S1 levels (J-L). The last follow-up MRI grade was A at the $L 3 / 4, L 4 / 5$, and $L 5 / S 1$ levels. Figure is available in color online only.

implants. In addition, enough distraction and keeping the cage back as much as possible are crucial to obtain the central canal to a greater degree. We elevated both legs to a backward-keeping prone position, and maximum rod compression was performed to obtain more lordosis.

Indirect spinal canal decompression is a process used to reduce disc bulging, extend the hypertrophied ligamentum flavum, and restore disc height. ${ }^{4}$ The height of the cage is an important component of the degree of indirect decompression. Because the anterior longitudinal ligament is cut during $\mathrm{ALIF}^{23}$ and a tall cage is inserted, increased distraction in the canal and foramen can theoretically be achieved. Additionally, direct removal of disc is also a process for improving indirect canal decompression. According to Ohtori et al., ${ }^{34}$ at the 10-year follow-up after ALIF, the average cross-sectional area of the ligament flavum at the operated level was significantly smaller than it was preoperatively, and the DSCSA was significantly expanded at fusion sites compared with nonfusion sites. The stability of the spine may have caused the change in the lumbar ligamentum flavum and remodeling of the spinal canal. ${ }^{34}$ In our study, there was more improvement for spinal stenosis demonstrated on the last follow-up MRI than on the postoperative MRI. We believe that remodeling leading to a decrease in the degenerative changes that cause stenosis (disc bulge, ligamentum and facet capsule hypertrophy) is a more important process than restoring the disc height and direct disc removal in indirect decompression while fusion is performed successfully.

The strength of the present study is that this is the first quantitative analysis of indirect decompression after ALIF for central spinal canal stenosis. Only one study has shown indirect decompression of the central spinal canal after ALIF, but that study was limited to 10 patients and did not use preoperative MRI scans, only postoperative and follow-up MRI scans. ${ }^{34}$ In our study, we obtained preop- 
erative, postoperative, and last follow-up MRI in patients undergoing ALIF to measure the extent of central spinal canal decompression at each spinal level. This study provides a comparison of the preoperative and postoperative measurements of the central spinal canal after decompression with ALIF in 64 patients at 123 spinal fusion levels to overcome errors due to too little data and to obtain reliable results. In our study, we applied a sensitive grading system (grades A-C) to classify stenosis based on the morphology and density of rootlets in the CSF on high-resolution MRI. The extent of decompression after surgery was objectively determine $\mathrm{d}^{13}$ using the DSCSA as a more sensitive parameter representing lumbar central spinal canal stenosis than the spinal canal cross-sectional area. ${ }^{35}$

\section{Limitations}

This study has several limitations. First, although this study is not a very small case series with a limited number of patients, further studies with a larger sample size may be necessary to verify the results. Second, the heterogeneity of the techniques and the variability in the surgeon's experience are additional limitations. This study may reflect the technical skill of the surgeon involved, and the findings may not be generalizable. Third, there is no control group that was treated with an alternative interbody fusion surgery for a specific comparison; however, we found excellent results in our study. More studies, especially randomized controlled trials and studies with control groups, are needed to further explore this topic. Fourth, we performed MRI on the day before surgery, on postoperative day 2, and on the last follow-up day after surgery. Patients without data after 12 months were excluded, so all final results were categorized as those obtained more than 12 months after surgery. However, the final follow-up time was different for each patient and could affect the outcome. It is considered that the error in results could be reduced if the follow-up time after surgery was applied equally to each patient.

Nevertheless, this is the first study to quantitatively analyze indirect decompression after ALIF for lumbar spinal stenosis. The findings could serve as a reference in the assessment of indirect decompression for central spinal canal stenosis via ALIF, but further research should be conducted.

\section{Conclusions}

The ALIF approach can be a reasonable alternative to extensive posterior approaches. We suggest that the ALIF procedure can be used for decompression in central spinal canal stenosis to restore the foraminal dimensions, thus allowing decompression of the nerve roots. In patients with multilevel central spinal canal stenosis requiring spinal fusion, ALIF can be a good minimally invasive choice to achieve indirect decompression, avoiding the need for the direct resection of posterior elements.

\section{References}

1. Mobbs RJ, Phan K, Malham G, et al. Lumbar interbody fusion: techniques, indications and comparison of interbody fusion options including PLIF, TLIF, MI-TLIF, OLIF/ATP, LLIF and ALIF. J Spine Surg. 2015;1(1):2-18.
2. Teng I, Han J, Phan K, Mobbs R. A meta-analysis comparing ALIF, PLIF, TLIF and LLIF. J Clin Neurosci. 2017;44:11-17.

3. Elowitz EH, Yanni DS, Chwajol M, et al. Evaluation of indirect decompression of the lumbar spinal canal following minimally invasive lateral transpsoas interbody fusion: radiographic and outcome analysis. Minim Invasive Neurosurg. 2011;54(5-6):201-206.

4. Fujibayashi S, Hynes RA, Otsuki B, et al. Effect of indirect neural decompression through oblique lateral interbody fusion for degenerative lumbar disease. Spine (Phila Pa 1976). 2015;40(3):E175-E182.

5. Janssen I, Lang G, Navarro-Ramirez R, et al. Can fan-beam interactive computed tomography accurately predict indirect decompression in minimally invasive spine surgery fusion procedures? World Neurosurg. 2017;107:322-333.

6. Oliveira L, Marchi L, Coutinho E, Pimenta L. A radiographic assessment of the ability of the extreme lateral interbody fusion procedure to indirectly decompress the neural elements. Spine (Phila Pa 1976). 2010;35(26 Suppl):S331-S337.

7. Sato J, Ohtori S, Orita S, et al. Radiographic evaluation of indirect decompression of mini-open anterior retroperitoneal lumbar interbody fusion: oblique lateral interbody fusion for degenerated lumbar spondylolisthesis. Eur Spine J. 2017; 26(3):671-678.

8. Januszewski J, Beckman JM, Bach K, et al. Indirect decompression and reduction of lumbar spondylolisthesis does not result in higher rates of immediate and long term complications. J Clin Neurosci. 2017;45:218-222.

9. Kapustka B, Kiwic G, Chodakowski P, et al. Anterior lumbar interbody fusion (ALIF): biometrical results and own experiences. Neurosurg Rev. 2020;43(2):687-693.

10. Rao PJ, Maharaj MM, Phan K, et al. Indirect foraminal decompression after anterior lumbar interbody fusion: a prospective radiographic study using a new pedicle-to-pedicle technique. Spine J. 2015;15(5):817-824.

11. Shin SH, Choi WG, Hwang BW, et al. Microscopic anterior foraminal decompression combined with anterior lumbar interbody fusion. Spine J. 2013;13(10):1190-1199.

12. Hayama S, Nakano A, Nakaya Y, et al. The evaluation of indirect neural decompression after lateral lumbar interbody fusion using intraoperative computed tomography myelogram. World Neurosurg. 2018;120:e710-e718.

13. Schizas C, Theumann N, Burn A, et al. Qualitative grading of severity of lumbar spinal stenosis based on the morphology of the dural sac on magnetic resonance images. Spine (Phila Pa 1976). 2010;35(21):1919-1924.

14. Fleiss JL. Measuring nominal scale agreement among many raters. Psychol Bull. 1971;76(5):378-382.

15. Zanoli G, Strömqvist B, Jönsson B. Visual analog scales for interpretation of back and leg pain intensity in patients operated for degenerative lumbar spine disorders. Spine (Phila Pa 1976). 2001;26(21):2375-2380.

16. Fairbank JC, Pynsent PB. The Oswestry Disability Index. Spine (Phila Pa 1976). 2000;25(22):2940-2952.

17. Macnab I. Negative disc exploration. An analysis of the causes of nerve-root involvement in sixty-eight patients. $J$ Bone Joint Surg Am. 1971;53(5):891-903.

18. Rao PJ, Loganathan A, Yeung V, Mobbs RJ. Outcomes of anterior lumbar interbody fusion surgery based on indication: a prospective study. Neurosurgery. 2015;76(1):7-24.

19. Hsieh PC, Koski TR, O'Shaughnessy BA, et al. Anterior lumbar interbody fusion in comparison with transforaminal lumbar interbody fusion: implications for the restoration of foraminal height, local disc angle, lumbar lordosis, and sagittal balance. J Neurosurg Spine. 2007;7(4):379-386.

20. Malham GM, Parker RM, Ellis NJ, et al. Anterior lumbar interbody fusion using recombinant human bone morphogenetic protein-2: a prospective study of complications. J Neurosurg Spine. 2014;21(6):851-860. 
21. Mobbs RJ, Phan K, Thayaparan GK, Rao PJ. Anterior lumbar interbody fusion as a salvage technique for pseudarthrosis following posterior lumbar fusion surgery. Global Spine $J$. 2016;6(1):14-20.

22. Phan K, Thayaparan GK, Mobbs RJ. Anterior lumbar interbody fusion versus transforaminal lumbar interbody fusionsystematic review and meta-analysis. Br J Neurosurg. 2015; 29(5):705-711.

23. Yoshihara H. Indirect decompression in spinal surgery. J Clin Neurosci. 2017:44:63-68.

24. Cho W, Sokolowski MJ, Mehbod AA, et al. MRI measurement of neuroforaminal dimension at the index and supradjacent levels after anterior lumbar interbody fusion: a prospective study. Clin Orthop Surg. 2013;5(1):49-54.

25. Rao PJ, Ghent F, Phan K, et al. Stand-alone anterior lumbar interbody fusion for treatment of degenerative spondylolisthesis. J Clin Neurosci. 2015;22(10):1619-1624.

26. Gumbs AA, Bloom ND, Bitan FD, Hanan SH. Open anterior approaches for lumbar spine procedures. Am J Surg. 2007; 194(1):98-102.

27. Shim JH, Kim WS, Kim JH, et al. Comparison of instrumented posterolateral fusion versus percutaneous pedicle screw fixation combined with anterior lumbar interbody fusion in elderly patients with L5-S1 isthmic spondylolisthesis and foraminal stenosis. J Neurosurg Spine. 2011;15(3):311-319.

28. Strube P, Hoff E, Hartwig T, et al. Stand-alone anterior versus anteroposterior lumbar interbody single-level fusion after a mean follow-up of 41 months. J Spinal Disord Tech. 2012; 25(7):362-369.

29. Hrabalek L, Adamus M, Gryga A, et al. A comparison of complication rate between anterior and lateral approaches to the lumbar spine. Biomed Pap Med Fac Univ Palacky Olomouc Czech Repub. 2014;158(1):127-132.

30. Laws CJ, Coughlin DG, Lotz JC, et al. Direct lateral approach to lumbar fusion is a biomechanically equivalent alternative to the anterior approach: an in vitro study. Spine (Phila Pa 1976). 2012;37(10):819-825.

31. Malham GM, Parker RM, Blecher CM, et al. Choice of approach does not affect clinical and radiologic outcomes: a comparative cohort of patients having anterior lumbar interbody fusion and patients having lateral lumbar interbody fusion at 24 months. Global Spine J. 2016;6(5):472-481.
32. Watkins RG IV, Hanna R, Chang D, Watkins RG III. Sagittal alignment after lumbar interbody fusion: comparing anterior, lateral, and transforaminal approaches. J Spinal Disord Tech. 2014;27(5):253-256

33. Malham GM, Parker RM, Goss B, et al. Indirect foraminal decompression is independent of metabolically active facet arthropathy in extreme lateral interbody fusion. Spine (Phila Pa 1976). 2014;39(22):E1303-E1310.

34. Ohtori S, Orita S, Yamauchi K, et al. Change of lumbar ligamentum flavum after indirect decompression using anterior lumbar interbody fusion. Asian Spine J. 2017;11(1):105-112.

35. Lim YS, Mun JU, Seo MS, et al. Dural sac area is a more sensitive parameter for evaluating lumbar spinal stenosis than spinal canal area: a retrospective study. Medicine (Baltimore). 2017;96(49):e9087.

\section{Disclosures}

The authors report no conflict of interest concerning the materials or methods used in this study or the findings specified in this paper.

\section{Author Contributions}

Conception and design: DH Lee. Acquisition of data: DH Lee, DG Lee, Hwang. Analysis and interpretation of data: DH Lee, DG Lee, Hwang. Drafting the article: DH Lee, DG Lee, Jang. Critically revising the article: DH Lee, DG Lee. Reviewed submitted version of manuscript: Park, DH Lee, Jang. Approved the final version of the manuscript on behalf of all authors: Park. Statistical analysis: DH Lee, DG Lee. Administrative/technical/material support: Park, Maeng. Study supervision: Park.

\section{Correspondence}

Choon Keun Park: Spine Center, The Leon Wiltse Memorial Hospital, Gyeonggi-do, South Korea. allspine@wiltse.co.kr. 